arranged were establishing restrictions and limitations, adjudication, establishing causation, optimising treatment, determining maximum medical recovery, providing a diagnosis, establishing a prognosis, and delaying claim payment. A number of important limitations to the current approach to IMEs were identified, including lack of specific training or accreditation for clinicians performing these assessments, lack of standards for assessment reports, and the potential for considerable conflict of interest in that referral sources select clinicians that are well paid to provide reports, and sent more referrals if their reports are valued.

Conclusions Our review provides guidance on priorities for future research regarding the conduct, use, and interpretation of IMEs.

\section{SOCIAL SECURITY IN BRAZIL: THE IMPACT OF EPIDEMIOLOGICAL NEXUS ON THE BENEFITS RELATED TO OCCUPATIONAL DISEASES}

C A S Salim. Fundacentro/Ministry of Labour and Employment, Belo Horizonte, Brazil

10.1136/oemed-2013-101717.151

Objectives The Ministry of Social Security, in order to face the under-reporting of occupational accidents and diseases, has introduced new methods of identifying them. In addition to the Employment Accident Notification (CAT), the Technical Epidemiological Social Welfare Nexus (NTEP) was established in 2007. This work intends to analyse the role of the NTEP in improving information regarding to the work-related diseases and, more specifically, on the trend and variation of benefits paid for them.

Methods Through the NTEP it is possible to establish a nexus for each area of the economic activity, focusing on data of incapacitating diseases recognised by social welfare and involving more than 15 days' absence from work, using the ICD-10.

Results Benefits for work-related diseases increased 128.2\% during 2005-2008. However, the greatest changes occurred after 2007. From May 2006 to March 2007, when only the employer's CAT was used, 125,246 Accident and Disease Assistance authorisations were issued. But, with the addition of the NTEP to the CAT this number rose to 293,912 , an increase of $134.7 \%$ over the period from April 2007 to February 2008. The detachable figures are for 'musculoskeletal system and connective tissue' (107,764 cases), 'mental and behavioural disorders' (8,930 cases), and 'diseases of the nervous system' (8,396 cases).

Conclusions The accident benefits for work-related diseases are growing more than other welfare benefits. This reality requires more studies and technical insights as well as priorities in terms of specific strategies for OSH policy.

\section{SITUATION AND FACTORS RELATED TO HEALTH EXAMINATION ACCORDING TO OCCUPATIONAL RISK IN THAI GOVERNMENT HOSPITALS}

Ekpanyaskul, Noppakunphusit, Bundhukul. Faculty of Medicine, Srinakharinwirot University, Bangkok, Thailand

\subsection{6/oemed-2013-101717.152}

Periodic medical examinations are typically carried out in the context of a general health examination and an occupational health examination. In Thailand, government hospitals are not required to provide occupational health examinations, because these hospitals do not fall under the protection of Thai labour law. Therefore, the objective of this study was to evaluate the current practice of occupational health examinations in Thai government hospitals. The survey was conducted in August, 2011. The self-administrated questionnaire was sent by post to each government hospital. The questionnaire included information on demographic data of the hospital, 14 selected factors, and the activities regarding occupational health examinations. There were 465 hospitals participating in this study. Both percentage and multiple logistic regressions were utilised for statistical analyses. The results revealed that $82.6 \%$ (384/465) of Thai government hospitals conducted health examinations based on occupational risks to their healthcare workers. The uses that hospitals made of the results from their occupational health examinations were, from highest to lowest percentage, 93\% (357/384) to compare to previous results, $82 \%(315 / 384)$ to use in OHS activities, $48.7 \%(187 / 384)$ to use for hospital accreditation, and $15.6 \%(60 / 384)$ to conduct research. The factors of significance related to the health examinations in their hospitals were as follows: medium-sized hospitals (adjusted odds ratio $=8.96 ; 95 \%$ $\mathrm{CI}=2.04-39.30)$, the OHS support from the hospital's administration (adjusted odds ratio $=3.60 ; 95 \% \mathrm{CI}=1.69-7.65$ ), and OHS risk assessment activity (adjusted odds ratio $=4.87$; $95 \% \mathrm{CI}=2.28-10.41)$. In conclusion, hospitals where periodic occupational health examinations are not given as well as other relevant parties should initiate strategies to develop this examination for their healthcare workers to ensure their good health. Hospitals already providing this service should carefully consider how they use the data from their occupational examinations to raise their cost-effectiveness.

\section{HEALTH ASSESSMENT FOR WORKERS PERFORMING VISUAL EXAMINATION IN AN ELECTRONIC ENTERPRISE OF TAIWAN}

${ }^{1}$ P C Chu, ${ }^{2}$ Fuh. ${ }^{1}$ Taipei, Taiwan; ${ }^{2}$ Hwan-Ran Fuh, Taipei, Taiwan

10.1136/oemed-2013-101717.153

Objectives It is understood that visual display unit (VDU) workers complain of visual fatigue during or after work hours. The objective of the study is to conduct a cross-sectional health assessment for worker performing visual examination on electronic components.

Methods The 21 female workers performing visual examination on electronic component using microscopes in an electronic enterprise of Taiwan were the study population. The face to face questionnaire survey was conducted by the occupational health physician in 2011, and the content of questionnaire included demographic data, refractive disorders, subjective symptoms, and workplace risk factors related to visual health.

Results The mean age and years of employment of the study population were 31.5 and 2.9 years, respectively. The average period of visual examination was 10.5 hours per shift and poor work-rest schedule was noted. The average actual sleeping time was 6.8 hours per day. There were $36.4 \%$ and $31.8 \%$ workers needed to shift work and night work, respectively. $77.3 \%$ workers complained of visual symptoms. $90.9 \%$ workers had extra-ocular symptoms, and shoulder discomfort was the most common one of them $(81.8 \%)$. The workers with refractive disorders had higher visual fatigue than no disorders $(\mathrm{p}<$ 0.05). The workers with shift or night work patterns had higher both visual and extra-ocular symptoms than non-shift or non-night $(\mathrm{p}<0.05)$. The worker with visual fatigue had 